Gynäk. Rdsch. 1984;24(suppl. 1):I-III

\title{
Contents, Vol. 24, Supplement 1, 1984
}

\section{Index}

1. Hauptthema $\cdot$ ler sujet principal

Der habituelle Abort

Les avortements spontanés à repetition

Rundtischgespräch — Table ronde Moderator · Modérateur: W.P. Faulk, Nice

Maroni, E.: Der nicht immunologisch bedingte habituelle Abort 1

Bischof, P.; Herrmann, W.L.: Are Circulating Beta-2-Microglobulin Levels a Marker

of Feto-Maternal Histocompatibility?

9

2. Hauptthema $\square$ 2e sujet principal Reelle und falsche Risiken in der Kontrazeption Les risques

en contraception: vrais et faux

Rundtischgespräch — Table ronde Moderator — Modérateur: C. Revaz, Lausanne

Belaïsch, J.: Risques vrais et faux de la contraception hormonale orale

Revaz, C: Risques vrais et faux de la contraception hormonale injectable 20

Zufferey, M.-M.: Risques vrais et faux de la contraception locale: spermicides, diaphragme

Revaz, C: Risques vrais et faux des appareils intra-utérins

Zufferey, M.-M.: Risques vrais et faux des méthodes naturelles de planification des naissances

Index II

Zufferey, M.-M.: Risques des nouvelles méthodes en contraception 40

Belaïsch, J.: Risques vrais et faux de la contraception masculine 43

Béran, M.-D.: Risques vrais et faux de $\Gamma$ information contraceptive 49

3. Hauptthema $\cdot 3$ e sujet principal

Aktuelle Behandlung des Mammakarzinoms

Traitement actuel du cancer du sein

Rundtischgespräch — Table ronde

Moderator · Modérateur: W.E. Schreiner, Zurich

Schreiner, W.E.: Taktik und operatives Vorgehen bei der Behandlung des Mamma karzinoms 54

Hofmann, V.; Martz, G.: Chemotherapie des primären und metastasierenden Mam makarzinoms 78

Amalric, R.; Ayme, Y.; Brandone, H.; Spitalier, J.M.: Radiotherapie curative après chirurgie conservatrice des cancers du sein opérables

4. Hauptthema $\cdot 4 \mathrm{e}$ sujet principal

Neue Aspekte in der Geburtsleitung

Nouveaux aspects de la conduite de $\Gamma$ accouchement

Rundtischgespräch — Table ronde Moderator · Modérateur: E. Dreher, Bern

Richter, R.: Biophysikalisches und biochemisches fetales Monitoring

Stucki, D.: Quelques aspects de la conduite de la grossesse et de Гaccouchement 
gémellaires

108

Dreher, E.: Leitung der Frühgeburt

116

Sidiropoulos, D.: Mortalität und Morbidität der Frühgeborenen

5. Hauptthema $\cdot 5$ e sujet principal

Totale Hysterektomie: Indikationsstellung und Missbräuche Hysterectomies totales: indications

et abus

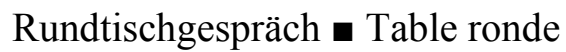

Moderator · Modérateur: E. Hochuli, Münsterlingen

Hochuli, E.: Totale Hysterektomie: Indikationsstellung und Missbräuche. Allge-

meine und schweizerische Aspekte 131

Lüscher, K.P.; Schneitter, J.; Vogt, H.P.; Künsch, U.: Hysterektomieverhalten an

einem Kantonsspital. Bericht über 3000 Hysterektomien an der Frauenklinik

Münsterlingen in den Jahren 1969-1983 140

Index

III

6. Havptthema $-6 e$ sujet principal Schwangerschaft und Geburt nach dem 40. Altersjahr Grossesse et accouchement après 40 ans

Rundtischgespräch - Table ronde Moderator · Modérateur: F. Béguin, Geneve

Béguin, F.: Introduction: age et organisme gravide 148

Siegrist, J.: Die spate Erstgebärende-Sozialmedizinische Aspekte 154

Autorenregister

159

Schweizerische Gesellschaft für Gynäkologie und Geburtshilfe Société Suisse de Gynécologie et Obstétrique Bericht über die Jahresversammlung, 1. bis 4. April 1984 in St. Moritz Rapport de

PAssemblée annuelle, 1 au 4 avril 1984 à St. Moritz

Redaktor — Rédacteur: E. Dreher, Bern ISBN 3-8055-3964-9 